\title{
Uma Abordagem Analítica da Codificação Turbo
}

\author{
Carolina A. Trigilio, Ivan R. S. Casella \\ Universidade Federal do $A B C$ \\ Centro de Engenharia, Modelagem e Ciências Sociais Aplicadas
}

\begin{abstract}
Resumo- Este artigo apresenta uma análise algébrica do processo de codificação e decodificação turbo, considerando inicialmente apenas uma iteração, para descrever o funcionamento dos algoritmos utilizados e para verificar sua capacidade de correção de erros.
\end{abstract}

Palavras-chave: turbo, codificação, FEC.

\section{Introdução}

A codificação turbo é uma estratégia de controle de erros que está sendo cada vez mais utilizada, já que aumenta a confiabilidade da informação que está sendo transferida, permitindo atingir índices de capacidade muito próximos das predições de Shannon [1]. Neste trabalho será apresentada uma metodologia algébrica de codificação e decodificação turbo baseada no algoritmo MAP (Maximum a Priori). O método apresentado será aplicado num sistema acústico de transmissão digital de imagens para reduzir a taxa de erro de bit (BER).

\section{Codificação}

O processo de codificação turbo utilizado neste artigo para a transmissão da mensagem $\mathbf{x}_{b}$, descrito na Figura 1, emprega a concatenação de dois codificadores convolucionais recursivos sistemáticos (RSC) por meio de entrelaçadores (П), de forma que a seqüência codificada $\mathbf{x}_{c}$ resultante é suficientemente aleatória para prover taxas de erro relativamente baixas, quando associado à técnicas iterativas de decodificação baseadas em informação suave [2].

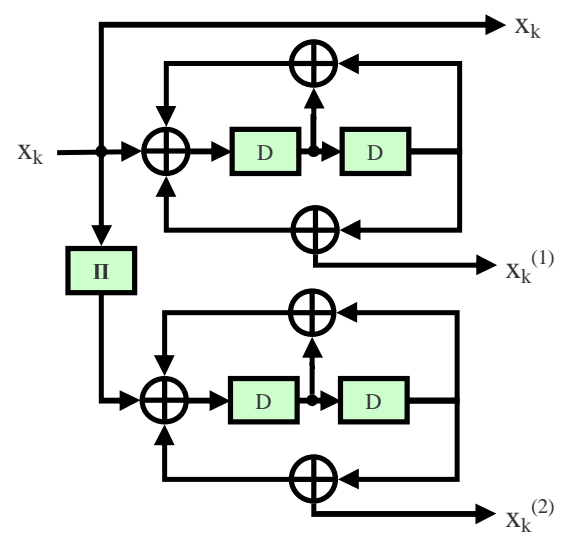

Figura 1- Modelo do codificador turbo de referência.

A seqüência codificada $\mathbf{x}_{c}$ é composta por $N$ palavras de código dadas por $\left(x_{k}, x_{k}{ }^{(1)}, x_{k}{ }^{(2)}\right)$, onde o bit de informação no instante $k, x_{k}$, pode assumir os valores -1 ou +1 com uma probabilidade a priori $P\left(x_{k}\right)$. Associada a esta probabilidade pode-se definir a LLR (Log Likelihood ratio) [3]:

$$
L\left(x_{k}\right)=\ln \left(\frac{P\left(x_{k}=+1\right)}{P\left(x_{k}=-1\right)}\right)
$$

Considerando que a seqüência $\mathbf{x}_{c}$ seja transmitida por um canal AWGN (Additive White Gaussian Noise), a sequiência recebida, $\mathbf{y}_{c}$, composta por palavras de código corrompidas por ruído representadas por $\left(\mathrm{y}_{\mathrm{k}}, \mathrm{y}_{\mathrm{k}}{ }^{(1)}, \mathrm{y}_{\mathrm{k}}{ }^{(2)}\right)$, é submetida ao processo de decodificação para recuperar os dados originais.

\section{Decodificação}

A decodificação pode ser realizada através de uma implementação iterativa do algoritmo MAP [3]. Na Figura 2, é apresentado o modelo de decodificação turbo utilizado nesse trabalho.

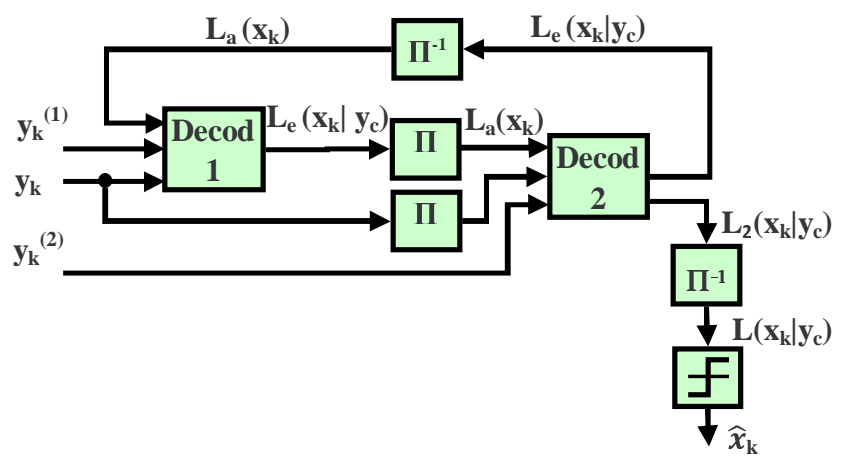

Figura 2- Decodificação Turbo.

No processo de decodificação iterativo é utilizada, além da informação recebida pelo canal, a informação intrínseca $a$ priori (API), denominada de $L_{a}\left(x_{k}\right)$, que é resultante do processo de decodificação anterior. A informação trocada entre os decodificadores, chamada de informação extrínseca e denominada de $L_{e}\left(x_{k} \mid \boldsymbol{y}_{c}\right)$, é a informação fornecida pelo processo de decodificação levando em consideração a seqüência total recebida e a API de todos os outros bits, excluindo a API do bit a ser decodificado [2].

O decodificador utiliza o algoritmo MAP [1] para estimar a sequiência de bits originais $\mathbf{x}_{b}$ através do cálculo da LLR $a$ posteriori $\mathrm{L}\left(x_{k} \mid \mathbf{y}_{\mathbf{c}}\right)$, dada por [3]:

$$
L\left(x_{k} \mid \mathbf{y}_{c}\right)=\ln \left(\frac{P\left(x_{k}=+1 \mid \mathbf{y}_{c}\right)}{P\left(x_{k}=-1 \mid \mathbf{y}_{c}\right)}\right)=\ln \left(\frac{\sum_{R 1} P\left(s^{\prime}, s, \mathbf{y}_{c}\right)}{\sum_{R 0} P\left(s^{\prime}, s, \mathbf{y}_{c}\right)}\right)
$$

Onde, $P\left(s^{\prime}, s, \mathbf{y}_{\boldsymbol{c}}\right)$ representa a probabilidade conjunta de no instante $k-1$ estar no estado da treliça $s^{\prime}$, no instante $k$ estar no estado da treliça $s$ e da seqüência recebida ser $\mathbf{y}_{\boldsymbol{c}}$.

A $P\left(s^{\prime}, s, \mathbf{y}_{c}\right)$ pode ser calculada iterativamente, fazendo [3]:

$$
P\left(s^{\prime}, s, \mathbf{y}_{c}\right)=\alpha_{k-1}\left(s^{\prime}\right) \cdot \gamma_{k}\left(s^{\prime}, s\right) \cdot \beta_{k}(s)
$$

Onde, 


$$
\begin{aligned}
& \gamma_{k}\left(s^{\prime}, s\right)=e^{\frac{x_{k} \cdot L_{a}\left(x_{k}\right)+2 \cdot x_{k} \cdot y_{k}+2 \cdot x_{k}^{(i)} \cdot y_{k}^{(i)}}{2}}, i=1,2 \\
& \alpha_{k}(s)=\sum_{s^{\prime}} \alpha_{k-1}\left(s^{\prime}\right) \cdot \gamma\left(s^{\prime}, s\right) \\
& \beta_{k-1}\left(s^{\prime}\right)=\sum_{s} \gamma_{k}\left(s^{\prime}, s\right) \cdot \beta_{k}(s)
\end{aligned}
$$

\section{Resultados Analíticos}

Nessa seção será apresentado um exemplo de cálculo analítico do processo de codificação e decodificação turbo empregando o algoritmo MAP.

Considerando o codificador [1 5/7] apresentado na Figura 1, pode-se obter a treliça de estados apresentada na Figura 3:

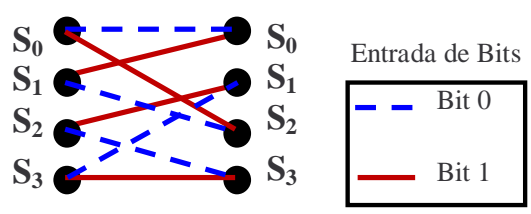

Figura 3- Treliça de Estados do codificador [1 5/7]

Na Tabela 1 são apresentados os estados atuais e futuros e os

\begin{tabular}{|c|c|c|c|c|}
\hline & \multicolumn{4}{|c|}{ Entrada (x) } \\
\hline Atual & & $\mathbf{0}$ & & 1 \\
\hline $\mathbf{S}_{\mathbf{0}}$ & $\mathbf{S}_{\mathbf{0}}$ & $Y_{1}(00)$ & $\mathbf{S}_{2}$ & $Y_{5}(11)$ \\
\hline $\mathbf{S}_{1}$ & $\mathbf{S}_{2}$ & $Y_{2}(00)$ & $S_{0}$ & $Y_{6}(11)$ \\
\hline $\mathbf{S}_{2}$ & $\mathbf{S}_{3}$ & $Y_{3}(01)$ & $\mathbf{S}_{1}$ & $Y_{7}(\mathbf{1 0})$ \\
\hline $\mathbf{S}_{3}$ & $\mathbf{S}_{1}$ & $Y_{4}(01)$ & $\mathbf{S}_{3}$ & $Y_{8}(10)$ \\
\hline
\end{tabular}
bits de saída produzidos pelos codificadores 1 e 2 :

Tabela 1: Estados e bits de entrada.

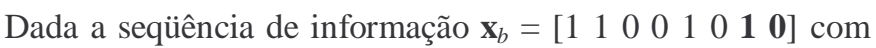
terminação e, considerando que o entrelaçador seja representado por $\Pi=\left[\begin{array}{llllllll}7 & 5 & 1 & 2 & 4 & 3 & 6 & 0\end{array}\right]$, tem-se a seguinte sequiência de transição de estados para o codificador 1:

$$
\stackrel{y 5}{S_{0}} \stackrel{\text { y7 }}{\rightarrow} S_{2} \stackrel{y 2}{\rightarrow} S_{1} \stackrel{\text { y3 }}{\rightarrow} S_{2} \stackrel{\text { y8 }}{\rightarrow} S_{3} \stackrel{\text { y4 }}{\rightarrow} S_{3} \stackrel{\text { y6 }}{\rightarrow} S_{1} \stackrel{y 1}{\rightarrow} S_{0} \stackrel{\rightarrow}{\rightarrow} S_{0}
$$

De forma semelhante, a sequiência de transição de estados para o codificador 2 é dada por:

$$
\begin{aligned}
& \begin{array}{llllllll}
y 1 & y 1 & y 5 & y 3 & y 8 & y 4 & y 6 & y 5
\end{array} \\
& s_{0} \rightarrow s_{0} \rightarrow s_{0} \rightarrow s_{2} \rightarrow s_{3} \rightarrow s_{3} \rightarrow s_{1} \rightarrow s_{0} \rightarrow s_{2}
\end{aligned}
$$

Deste modo, a seqüência codificada $\mathbf{x}_{c}$, utilizando uma representação polar $(+1$ e -1$)$, é dada por:

$$
\mathbf{x}_{\mathrm{c}}=[+1+1-1+1-1-1-1-1+1-1+1+1+1-1-1-1+1+1+1+1+1-1-1+1]
$$

Após a transmissão do código através de um canal AWGN com $\mathcal{N}(0,1)$, a sequiência recebida é dada por:

$$
\begin{aligned}
& \mathbf{y}_{\mathrm{k}}=\left[\begin{array}{llllllll}
2.96 & -0.232 & -0.249 & 0.83 & -0.26 & -0.79 & 0.43 & -0.74
\end{array}\right] \\
& \mathbf{y}_{\mathbf{k}}{ }^{(1)}=\left[\begin{array}{llllllll}
3.13 & -1.44 & -0.17 & 0.91 & -0.45 & 1.27 & 1.98 & -0.53
\end{array}\right] \\
& \mathbf{y}_{\mathbf{k}}^{(2)}=\left[\begin{array}{llllllll}
-1.70 & -1.69 & 1.82 & 2.04 & -3.05 & 1.46 & 2.10 & 0.30
\end{array}\right]
\end{aligned}
$$

A saída do decodificador 1 para a primeira iteração pode ser obtida através das fórmulas de $\alpha_{k}(s), \beta_{k}\left(s^{\prime}\right)$ e $\gamma_{k}\left(s^{\prime}, s\right)$, representadas nas Tabelas 2, 3 e 4, para $\alpha_{0}(0)=1$ e $\beta_{8}(0)=1$.
Tabela 2: Valores para $\alpha_{k}(s)$ para o Decodificador 1

\begin{tabular}{|l|c|c|c|c|c|c|c|c|c|}
\hline \multicolumn{10}{|c|}{ Tabela de $\boldsymbol{\alpha}$} \\
\hline $\mathbf{S}_{\mathbf{0}}$ & 1 & $5.0 \mathrm{e}^{-6}$ & $7.3 \mathrm{e}^{-6}$ & 0.27 & 0.08 & 0.12 & 0.12 & 0.61 & 0.64 \\
\hline $\mathbf{S}_{\mathbf{1}}$ & 0 & 0.99 & $2.6 \mathrm{e}^{-7}$ & 0.64 & 0.49 & 0.20 & 0.08 & 0.16 & 0.22 \\
\hline $\mathbf{S}_{\mathbf{2}}$ & 0 & 0 & 0.91 & 0.04 & 0.19 & 0.35 & 0.47 & 0.17 & 0.05 \\
\hline $\mathbf{S}_{\mathbf{3}}$ & 0 & 0 & 0.08 & 0.03 & 0.22 & 0.31 & 0.31 & 0.05 & 0.07 \\
\hline
\end{tabular}

Tabela 3: Valores para $\beta_{k}(s)$ para o Decodificador 1.

\begin{tabular}{|l|c|c|c|c|c|c|c|c|c|}
\hline \multicolumn{10}{|c|}{ Tabela de $\boldsymbol{\beta}$} \\
\hline $\mathbf{S}_{\mathbf{0}}$ & 0.29 & 0.56 & 0.49 & 0.62 & 0.01 & $8.0 \mathrm{e}^{-3}$ & $7.7 \mathrm{e}^{-3}$ & 0.92 & 1 \\
\hline $\mathbf{S}_{\mathbf{1}}$ & $5.0 \mathrm{e}^{-4}$ & 0.22 & 0.09 & 0.18 & 0.38 & 0.04 & $1.4 \mathrm{e}^{-3}$ & 0 & 0 \\
\hline $\mathbf{S}_{\mathbf{2}}$ & 0.70 & 0.12 & 0.31 & 0.03 & 0.04 & $1.6 \mathrm{e}^{-3}$ & 0.95 & 0.07 & 0 \\
\hline $\mathbf{S}_{\mathbf{3}}$ & $6.0 \mathrm{e}^{-4}$ & 0.08 & 0.08 & 0.15 & 0.55 & 0.95 & 0.03 & 0 & 0 \\
\hline
\end{tabular}

Tabela 4: Valores para $\gamma_{k}\left(s^{\prime}, s\right)$ para o Decodificador 1.

\begin{tabular}{|c|c|c|c|c|c|c|c|}
\hline \multicolumn{7}{|c|}{ Tabela de $\gamma$} \\
\hline 0.002 & 5.33 & 1.53 & 0.17 & 2.03 & 0.62 & 0.08 & 3.58 \\
\hline 445.0 & 0.18 & 0.65 & 5.71 & 0.49 & 1.60 & 11.1 & 0.27 \\
\hline 0.84 & 3.35 & 0.93 & 0.92 & 1.20 & 0.12 & 0.21 & 0.81 \\
\hline 1.18 & 0.29 & 1.07 & 1.08 & 0.83 & 7.87 & 4.70 & 1.23 \\
\hline 445.0 & 0.18 & 0.65 & 5.71 & 0.49 & 1.60 & 11.1 & 0.27 \\
\hline 0.002 & 5.33 & 1.53 & 0.17 & 2.03 & 0.62 & 0.08 & 3.58 \\
\hline 1.18 & 0.29 & 1.07 & 1.08 & 0.83 & 7.87 & 4.70 & 1.23 \\
\hline 0.84 & 3.35 & 0.93 & 0.92 & 1.20 & 0.12 & 0.21 & 0.81 \\
\hline
\end{tabular}

A partir dos valores de $\alpha, \beta$ e $\gamma$ pode-se calcular as probabilidades $P\left(s^{\prime}, s, \mathbf{y}_{c}\right)$ e a LLR resultante para o primeiro decodificador é dada por:

$$
\mathbf{L}_{\text {map } 1}=\left[\begin{array}{llllllll}
11.30 & 3.70 & 0.39 & 0.50 & -0.43 & -4.36 & 3.73 & -3.81
\end{array}\right]
$$

A seqüência de bits decodificada correspondente é dada por:

$$
\mathbf{x}_{1}=[+1+1+1+1-1-1+1-1]
$$

Usando um procedimento semelhante para o segundo decodificador, e considerando $\alpha_{0}(0)=1$ e $\beta_{8}(\mathrm{~s})=0.25$, tem-se:

$$
\mathbf{L}_{\text {map } 2}=\left[\begin{array}{llllllll}
-11.26 & -10.98 & 7.04 & -5.19 & 4.84 & -4.56 & 5.73 & 11.89
\end{array}\right]
$$

A seqüência de bits decodificada correspondente é dada por:

$$
\mathbf{x}_{2}=[-1-1+1-1+1-1+1+1]
$$

Após o desembaralhamento e conversão para bits (1 e 0), obtém-se a estimativa da mensagem original transmitida:

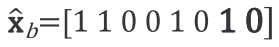

Pode-se notar que os erros ocorridos em $\mathbf{x}_{1}$ nos bits 2,3 e 4 foram corrigidos na saída $\mathbf{x}_{2}$.

\section{Conclusão}

Neste artigo foi apresentado um método analítico para a codificação e decodificação turbo. Pôde-se mostrar que a informação recebida, mesmo corrompida, contribui para a correção de erros, pois carrega uma probabilidade associada que aumenta em módulo a cada iteração devido ao entrelaçamento, que reduz a chance de erro no mesmo bit.

\section{Referências Bibliográficas}

[1] Haykin, Simon. Sistemas de Comunicação Analógicos e Digitais, Editora Bookman, $4^{\mathrm{a}}$ edição, 2004.

[2] Casella, Ivan R. S. Estruturas Espaço-Temporais de Múltiplas Entradas e Múltiplas Saídas para a Transmissão de Dados de Alta Taxa em Sistemas WCDMA, Teses de Doutorado, EPUSP, 2004.

[3] Abrantes, Silvio A. do Algoritmo BCJR à Descodificação Turbo, Relatório Técnico, FEUP, 2004. 